\title{
Los Telephone Book Papers de Franz Kline y su titubeante concordancia formal con la caligrafía japonesa
}

\author{
Iñigo Sarriugarte Gómez \\ Universidad del País Vasco
}

RESUMEN:

Franz Kline comenzó a ensayar sobre soportes que no eran habitualmente empleados en los procesos deductivos de los pintores expresionistas abstractos de la época, caso de las hojas de las guías telefónicas. El artista recabó un material de fácil uso y acceso, disponiendo de todo aquel que se necesitara, y de este modo poder eliminar los resultados no satisfactorios, ya que la disponibilidad de recursos era inagotable. También tuvo en consideración las reglamentaciones organizativas que aportaban las propias listas de teléfonos con la alineación de los nombres de los usuarios.

En base a una intensa revisión bibliográfica, se ha abordado un texto de carácter crítico-histórico con el objetivo de preponderar la importancia de los primeros procesos experimentales del pintor abstracto, al tratarse de indagaciones que se tornan fundamentales desde el punto de vista estratégico no sólo para la subsiguiente materialización de su obra más conocida, sino para dar salida al posterior debate gestado en torno a las supuestas influencias de la caligrafía japonesa en la conformación de sus trazos.

\section{PALABRAS CLAVE:}

Kline, papel de guías telefónicas, caligrafía, Expresionismo Abstracto.

\section{ABSTRACT:}

Franz Kline (1910-1962) started to dealt with supports that were not usually employed in the deductive processes of the painters of the time, for example the Telephone Books Papers. The artist collected a material of easy use and access, having all that was needed, and in this way to eliminate the unsatisfactory results, because of the availability of resources was inexhaustible. On the other hand, he studied the organizational regulations that provided the telephone lists themselves with the alignment of the names of the users.

Based on an intense bibliographical review, we have approached a text of critical-historical nature with the aim of preponderating the importance of the first experimental processes of the abstract painter, because these are questions that become fundamental from the strategic point of view not only for the subsequent materialization of his best known work, but also for the subsequent debate about the supposed influences of Japanese calligraphy on the contion of his lines.

\section{KEYWORDS:}

Kline, Telephone Book Papers, Calligraphy, Abstract Expressionism. 


\section{Breves referencias biográficas}

Franz Kline (1910-1962) estudió pintura en la Universidad de Boston entre 1931 y 1935, así como en el Art Students League de New York. Viajó dos años después por París y Londres, ampliando su formación en esta última capital en la Heatherly's School of Art. Después de su periplo europeo, se establece en Nueva York, donde el propio ambiente urbano inspira sus primeros óleos y acuarelas, articulando un estilo cercano al realismo de la Ashcan School, combinado con su admiración por Rembrandt, Goya, Manet y Sargent. Sus primeros años en esta ciudad fueron marcados por la dificultad profesional, realizando murales pintados en bares a la vez que vendía ilustraciones a revistas.

Será en 1946 cuando inicia su aproximación a la pintura abstracta, en una dirección paralela a las propuestas de Willem de Kooning y Robert Motherwell, donde la configuración articulada por líneas y planos comienza a orientarse hacia estructuras más caligráficas, caso de The Dancer (1946). No solo estuvo inspirado por las pinturas blancas y negras de Willem de Kooning de los años 1946-49, sino incluso por el aumento de escala en los formatos, tal y como lo empezó a describir Elaine de Kooning: "Un dibujo negro de cuatro por cinco pulgadas de una mecedora...... apareció con gigantescos trazos negros que erradicaban cualquier imagen, los trazos expandiéndose como entidades en sí mismos, ajenos a cualquier entidad, excepto a su propia existencia." ${ }^{1}$ Evidentemente, Willem de Kooning le había ayudado a ampliar la escala de sus bocetos y dibujos con el uso de un proyector Bell-Opticon en 1948, lo que permitió a Kline observar sus delineaciones en poderosos gestos abstractos, de hecho, "el reconocimiento público a Kline le llegó relativamente tarde (1950) en comparación con otros importantes pintores expresionistas abstractos (Jackson Pollock, Willem de Kooning, Mark Rothko, Clyfford Still). Es cierto que Kline no había pasado mucho tiempo progresando hacia la abstracción. El creó un trabajo representativo y relacionado con la Ashcan School hasta 1946 y sólo entonces comenzó a inclinarse hacia el trabajo abstracto. 1949 fue el año de la así llamada conversión completa hacia la abstrac-

KOONING, Elaine de, Franz Kline Memorial Exhibition, Gallery of Modern Art, Washington, 1962, p. 14. ción. Según se cuenta, de Kooning, un amigo de éste, le había prestado un proyector opaco Bell-Opticon a Kline. Cuando estuvieron juntos en el estudio del pintor de Kooning, éste comenzó a ampliar algunos de los pequeños bocetos de Kline y se dio cuenta de que podían funcionar como trabajos de gran formato." 2

En torno a 1950, la paleta de Kline se enfocaría en el uso del negro mediante gruesas y rugosas líneas pintadas con grandes brochazos sobre fondo blanco. Estas imágenes serían expuestas por primera vez en Charles Egan Gallery de New York durante ese mismo año. Después de esta muestra, alcanza un mayor prestigio, lo que le permitirá tomar parte en la colectiva The New American Painting (1958), celebrada en el Museo de Arte Moderno de New York, siendo exhibida posteriormente en ocho ciudades europeas. En estos momentos, Kline ya muestra la solvencia en el uso de un lenguaje propio, junto con la simplificación de la paleta al blanco y el negro.

La ampliación de los pequeños formatos en propuestas más monumentales, caso de $M a-$ honing (1956), llegaría a tener una relevante influencia en los escultores constructivistas de los años sesenta, especialmente potenciado por la constante equiparable del blanco y el negro, lo que permitía advertir un tipo de estructura compuesta por sólidos blancos en huecos negros. Por otro lado, la falta de interés por parte de Kline a la hora de atribuir mensajes ocultos en sus pinturas fue otro revulsivo para el análisis que llevaron a cabo escultores minimalistas como Donald Judd y Richard Serra. Para Matthew Israel: "Se ha comentado también que el trabajo de Kline debería ser observado dentro de la historia de la escultura constructiva americana, remontándose a David Smith. En este sentido, las esculturas de Richard Serra han sido llamadas como las made solid and three-dimensional de Kline y las formas arquitectónicas de éste probaron ser una inspiración para minimalistas como Donald Judd." 3

Por otro lado, durante los años 50, dio clases en conocidos centros artísticos, como Black Mountain College en North Carolina y el The Pratt Institute en Brooklyn. De hecho,

\footnotetext{
ISRAEL, Matthew; KLINE, Franz, "The Forgotten Abstract Expressionist?”, The Huffington Post, 8-12-2014. Web: http://www.huffingtonpost.com/matthew-israel/ a-call-for-a-newyork-fra_b_5947262.html. Última consulta: 04-06-2018.

$3 \quad$ Ibid.
} 
uno de sus estudiantes más famosos sería el pintor abstracto Cy Twombly, que generaría un estilo icónico propio en base a las técnicas enseñadas por Kline en 1951 en esta primera institución educativa.

En cualquier caso, entre las últimas muestras realizadas sobre la obra del artista, debemos anotar Franz Kline: arte y estructura de la identidad $^{4}$, donde no sólo se analizaban las distintas estructuras internas del estilo del artista, sino que a la vez se divulgaba la visión de un pintor que no trabajaba unilateralmente entre el blanco y el negro, ya que articulaba problemáticas en relación con el color, siendo explicada dicha cuestión por Kline de la siguiente manera: "No considero que el color sea un elemento añadido. Solo quiero sentirme libre de pintar en color o en blanco y negro. Yo pintaba originalmente en color y llegué al blanco y negro tapando el color. Luego empecé a pintar solo en color, con blanco y sin negro, y al final, en color y en blanco y negro. No persigo necesariamente el mismo objetivo con las distintas combinaciones. Aunque algunos digan que el blanco y negro es color, para mi, el color es otra cosa. En otras palabras, un área de azul intenso o la interrelación de dos colores distintos no es lo mismo que el blanco y negro. Cuando utilizo el color, nunca lo veo como un añadido o una forma de decorar la pintura en blanco y negro. Solo quiero sentirme libre de trabajar de ambas maneras. Y el hecho de que alguien utilice rosa, amarillo y rojo no le convierte forzosamente en colorista." ${ }^{5}$

Entre las ideas estéticas que estimularon a los expresionistas abstractos, incluido Franz Kline, encontramos los planteamientos de los surrealistas, así como el pensamiento de John Dewey, siendo publicado en 1934 bajo el título Art as Experience. La base de la estética de Dewey reside en su formulación de la experiencia artística, siendo tomada dicha práctica (opuesta a la experiencia normal) como un

Exposición realizada en el Museo Nacional Centro de Arte Reina Sofía (27 septiembre - 21 noviembre, 1994) de Madrid por Stephen C. Foster (Universidad de Iowa) y Manuel Borja-Villel (Fundación Tàpies). La propuesta tuvo el siguiente itinerario: Fundació Antoni Tàpies, Barcelona (18 marzo - 5 junio, 1994); Whitechapel Art Gallery, Londres (8 julio - 11 septiembre, 1994); Saarland Museum, Saarbrücken, Alemania (11 diciembre 5 febrero, 1994-95).

5 FUNDACIÓ ANTONI TẢPIES, Franz Kline: arte $y$ estructura de la identidad, Fundació Antoni Tàpies, Barcelona, 1994, p. 18. encuentro total con el fenómeno externo, lo que abarcaba desde el comienzo hasta el final y se integraba completamente en la conciencia como una entidad distinta de otras prácticas. La importancia de que Dewey se basara en la emoción sobre el intelecto era otro fundamento de su teoría que fortaleció la orientación procesual de estos pintores. De hecho, los sentimientos personales, no intelectuales, establecían el principal orden de las partes de la experiencia, seleccionando y discriminando el material que debía ser empleado por el artista.

Igualmente, resulta evidente la inclinación del Expresionismo Abstracto por el existencialismo y especialmente por el pensamiento de Soren Kierkegaard, siendo relevante el énfasis de este pensador por la condición trágica de la vida y su noción de libertad como un planteamiento subyacente de la desesperación. También, se acercaron al existencialismo fenomenológico de Jean-Paul Sartre, lo que les permitió consolidar una mayor sintonía entre estos creadores. Nancy Jachec ejercita una interesante reflexión sobre esta cuestión: "La intensidad de la prensa artística para definir el Expresionismo Abstracto americano como un arte existencialista se pudo comprobar mediante su habilidad para promocionar artistas con éxito anteriormente no asociados con el movimiento. Un ejemplo de esto fue la adquisición del artista Franz Kline previamente independiente de estatura internacional en la última mitad de los 50. Aunque Kline, un pintor gestual, había desarrollado su estilo caligráfico de signos en negro y blanco por 1947, el no participó en muchas de las actividades grupales de los expresionistas abstractos en aquel tiempo.”6

Para el poeta Frank 0'Hara, Kline era la quinta esencia de un action painter, revelándose sus pinturas en blanco y negro como referentes básicos para el establecimiento de una abstracción gestual y caligráfica dentro del Expresionismo Abstracto.

No debemos olvidar que Willem de Kooning y Franz Kline se acercaron filosóficamente a William Barrett, quien escribió What Is Existentialism?"; a la vez que este primero

\footnotetext{
6 JACHEC, Nancy, The Philosophy and Politics of $A b$ stract Expressionism, Cambridge University Press, Cambridge, 2000, p. 182.

7 BARRETT, William, What is Existentialism?, Grove Press, New York, 1964. La primera mitad del libro apareció originalmente publicada en la revista Partisan Review (1948).
} 
y Robert Motherwell ya se encontraban próximos a Harold Rosenberg, quien defendía estas conexiones estéticas. A finales de la década de 1940, el término "existencialismo" aparecía con frecuencia en las discusiones del $A r$ tist's $C l u b^{8}$, al igual que otros tantos términos como la ambigüedad y el compromiso. Otro referente de encuentro para los artistas del Expresionismo Abstracto fue el Eighth Street Club, asociación de artistas creada en otoño de 1949 por unos veinte miembros fundadores entre los que se encontraban Kline, Charles Egan, Willem de Kooning y Ad Reinhardt. Sería clausurado en primavera de 1962 y llevaron en la década de los años 50 toda una serie de mesas redondas, conferencias, intercambio de opiniones entre artistas, galeristas, escritores, directores de museos, etc. Esta especie de sociedad grupal también se solía reunir en el Waldorf Cafeteria, el Club, Studio 35 y la Cedar Tavern ${ }^{9}$, así como otros lugares del centro de Nueva York, lo que demuestra, a diferencia de lo que planteaba Rosenberg, el sentido de comunidad que tenían tales creadores. En el caso más concreto de Franz Kline, éste tuvo contactos profesionales con Jackson Pollock, Philip Guston y especialmente con Willem de Kooning, tal y como lo hemos comentado anteriormente, congregándose con dichos creadores en el downtown neoyorquino y ubicando su principal punto de encuentro en el mítico Cedar Tavern de Greenwich Village. No obstante, Kline quiso mantener una separación de intereses personales, ya que no abogó por el gesto ni la práctica pictórica sustentada en una aplicación catártica con el objetivo de liberar al yo interior del artista. En cambio, sus composiciones mostraban un mayor carácter arquitectónico, evocando puentes, edificios y estructuras arquitectónicas.

8 CLARKE, David James, The Influence of Oriental Philosophy on Postwar American Painting and Sculpture, The Courtauld Institute, London, 1988, p. 77.

9 Para obtener más información al respecto, consultar McDARRAH, Gloria, "The Club. The Artist's World in Pictures: The New York School", en The Artist's World in Pictures: The Photo Classic That Documents the New York School Action Painters, Shapolsky Publishers, New York, 1988, pp. 69-76; SANDLER, Irving, "The Club, How the Artists of the New York School Found Their First audience-Themselves", Artforum 4 (September 1965), p. 27; ALCOPLEY, Lewin, "The Club: The First Three Years", A Journal for Artists, 4 (fall 1985), pp. $45-47$.

\section{Procesos de trabajo sobre hojas de guías telefónicas}

Sin caer en una pintura de temas, aplicó el color en base a una articulación constructiva con dos energías antagónicas, priorizando la ejecución de bocetos y dibujos preparatorios, que serían articulados en base a papeles de periódicos y especialmente sobre hojas de guías telefónicas. Igualmente, se centró en analizar diversas cuestiones estructurales, como espacios, combinaciones de líneas, aplicación de ángulos, en un constante interés por el control ejecutivo de sus propuestas. Aparentemente, los trabajos están estructurados con una visible rigidez compositiva, pero también mantienen una evidente orientación gestual, donde el espectador no sólo debe observarlo a modo de símbolo lingüístico, sino que debe centrar la mirada en el efecto visual de profundidad logrado mediante acumulaciones de líneas verticales, horizontales y diagonales. Para numerosos autores ${ }^{10}$, estas hojas de guías telefónicas, cubiertas con huellas de pintura negra, se convirtieron en una de las principales fuentes de experimentación pictórica y compositiva, que posteriormente se materializarian en formatos de mayor escala sobre lienzo, lo que le darían un reconocimiento profesional.

Incluso, para T.B. Hess ${ }^{11}$, el uso de estos soportes se equipara al de un objet trouve que va a ser manipulado para todo tipo de prácticas, como limpiar un cepillo y observar su efecto sobre la hoja; colocar encima la tapa húmeda de un bote de pintura, quedando impresa dicha forma; experimentar con los grosores y cualidades físicas de cepillos y brochas; aplicar y hacer uso de coágulos de pintura vieja y seca; promover distintos derramamientos pictóricos más o menos controlados; reutilizarlas incluso con la existencia de otros elementos adheridos a dichas páginas, como la suciedad, manchas y otras sustancias no artísticas, que pudieran contener dichos formatos; incluso hasta podían aparecer restos de comida o alimentos consu-

\footnotetext{
10 HESS, Barbara, Expresionismo Abstracto, Taschen, Madrid, 2009, p. 84. Los trabajos realizados sobre dichos soportes están distribuidos por diferentes museos del mundo, así como en colecciones particulares. También, algunas de estas propuestas fueron destruidas por parte del artista. Se debe anotar que no hay datos o constancia del número de obras realizadas sobre dichos medios. 11 HESS, Thomas B., "Interview", New York Magazine, (April 7, 1972), s/n.
} 
midos, así como marcas de grasa o lamparones al ser envuelta la comida por el papel de periódico o de la guía telefónica. Kline aceptaba todas estas intrusiones físicas, como la propia composición estructural y cromática de las páginas amarillentas de la guía telefónica, lo que a su vez aportaba una pátina que inoculaba una sensación de antigüedad y de uso, quizás intentando plantear un acercamiento pigmentado a la seda empleada habitualmente en la pintura tradicional asiática.

Elaine de Kooning afirmaba lo siguiente: "En 1950, sus bocetos, medidos en pies en lugar de pulgadas, eran cortados de las páginas de las guías telefónicas y de hojas de periódico con esmalte negro" ${ }^{12}$. De acuerdo a la misma autora y según determinadas fotografías de época, se sabe que en su estudio no tenía grandes telas clavadas en las paredes, sino principalmente páginas de la guía telefónica repartidas y ubicadas por diferentes lugares. En una línea de similitud enunciativa, según Barbara Hess, "para realizar sus cuadros, Kline solía partir de dibujos espontáneos, realizados muchas veces por motivos económicos en las páginas de la guía telefónica o en papel de periódico."13

Debemos recordar que Kline había trabajado como dibujante de viñetas para el periódico del instituto, siendo quizás en estos momentos cuando se produce su contacto más técnico con las características físicas del papel. Posteriormente, derivaría a la realización de toda una serie de prácticas sobre los telephone book papers especialmente en tinta y óleo con una paleta reducida al negro y blanco, que más adelante tendría su repercusión en las propuestas con formatos de mayor escala y sobre lienzo, de hecho, Matthew Israel apunta lo siguiente: "Como los trabajos de Pollock y de Kooning, las pinturas de Kline expresan dinamismo, movimiento y expresión a gran escala, pero son también premeditadas, elaboradas en base a una elevada construcción. Estos no fueron solo rápidos e instintivos gestos. Cada línea era a menudo movida alrededor y se remodelaba hasta que Kline encontraba su composición ideal. También Kline no sólo pintaba negro sobre fondo blanco, sino que empleaba el negro y el blanco como positivos. En base a una mirada cercana, puedes ver como sus blancos no son sólo fondos, sino que son empleados activamente para

\footnotetext{
2 KOONING, opus. cit., p. 15.

13 HESS, Barbara, opus. cit., p. 84.
}

unirse con los negros. De esta manera, las capas tanto de negro y blanco permitían construir sus trabajos finales." 14

En cierta manera, su interés por el estudio de lo opuesto entre ambos colores no podía avanzar más con el constante uso de las páginas de las guías telefónicas, ya que estos soportes sólo mostraban fondos amarillentos; a la vez que el artista era consciente que el paso del tiempo amarillearía más la base de la hoja, así que tuvo irremediablemente que pasarse a trabajar sobre papeles de fondo blanco y lienzos.

Emmanuel Navaretta ${ }^{15}$, que compartió loft con Kline durante once meses en 1950, dice que en aquella época todo estaba basado en dibujos hechos en las páginas de las guías telefónicas. Kline usaba estas, en lugar de papel de dibujo, porque podían ser fácilmente encontradas. Robert Goldwater ha señalado que el patrón de impresión en estas páginas era "evitar cualquier recesión de perspectiva equivocada” ${ }^{16}$, convirtiéndose en otro motivo por el que a Kline le gustaba pintar sobre ellos. Debemos recordar que al emplear una página extraída de una guía telefónica el artista se beneficia de una estructura ordenada con secciones perfectamente articuladas y que podían servir como un soporte reglado donde ubicar sus diferentes propuestas compositivas.

Kline solía analizar cuidadosamente los dibujos realizados en las páginas telefónicas, que eran apilados por cientos en una esquina. El proceso de trabajo del artista asumía los siguientes pasos: después de seleccionar un dibujo que intuía que podía funcionar como un cuadro, entonces lo pegaba a un pedazo de cartón y se apuntalaba a un lado o era sujetado en la mano; de este modo, se podía observar de cerca, para posteriormente proceder a pintarlo directamente sobre el lienzo. Evidentemente no le gustaba transferir la imagen con ningún tipo de sistema de cuadrícula, lo que permitía producir variaciones respecto al original. Como excepción, la propuesta High Street (1950) puede haber pasado por una fase de rejilla como lo

\footnotetext{
14 ISRAEL, opus. cit., s/n.

15 NAVARETTA, Emmanuel. En GAUGH, Harry F., The Art of Franz Kline, 1930-1950. Figurative to Mature Abstraction, Indiana University, Fine Arts University Microfilms, A XEROX Company, Ann Arbor, Michigan, 1972, pp. 346-347.

16 GOLDWATER, Robert, "Introduction", en Franz Kline, 1910-1962: Exhibition Catalogue, Marlborough-Gerson Gallery, New York, 1967, p. 7.
} 
indica un dibujo en papel cuadriculado, a pesar de que comenzó como un croquis de página de guía telefónica. Incluso, se aprecia la influencia del Bell-Opticon, ya que la fuerte luz ayudaba a plasmar con más intensidad una gran imagen, quemando ligeramente algunas de las secciones de la página de la guía telefónica.

También, realizó sobre dichos medios, distintos collages, que indistintamente de su reducida escala, le permitía experimentar sobre la composición, pinceladas y materiales añadidos. Sobre estos collages ejecutados la mayoría de ellos a principios de los años cincuenta, solía aplicar varias veces óleo y témpera en la superficie empapelada, probando los gestos y las líneas caligráficas que más tarde se convertirían en su marca. Si bien en unos casos, Kline empleaba la página de la guía telefónica como fondo, en otros, como Untitled II (1952) (figura 1), cortaba estas páginas y las pegaba para desafiar la regularidad que caracteriza el diseño y la disposición gráfica de la guía telefónica. De este modo, consigue alterar la rigidez geométrica o la consistencia compositiva del formato original, yuxtaponiendo los cambios y las fracturas del collage con una pincelada que frecuentemente detiene o cambia de dirección. Kline adopta uno de los principales logros del collage: desplazar la continuidad del ilusionismo pictórico que caracterizó al arte desde el Renacimiento. Como muchos de los expresionistas abstractos, Kline parece haber considerado el componente del collage como análogo a la aplicación de la pintura. La compleja formación de capas de pigmento y corte de papel se produce en intervalos y sigue siendo difícil de discernir claramente el proceso exacto por el cual se realiza el trabajo.

Tanto en los collages como en el resto de propuestas sobre papel de guías telefónicas, según numerosos expertos, podemos observar lo siguiente: "Mientras que otros expresionistas abstractos están extrayendo sus propios sentimientos, intuiciones, emociones del subsconsciente y empleándolas para crear trabajos que son profundamente personales y plagados de sentidos ocultos, Kline hace un trabajo que versaba sobre las cualidades formales de la pintura, como el acto de pintar, el brochazo, composición y color." ${ }^{17}$ En este mismo sentido, la obra de Kline destaca por la técnica gestural icónica,

IDEELART, "Franz Kline and His Action Painting Manner", IdeelArt - The online gallerist for Contemporary Abstract Art. Web: http://www.ideelart.com/module/

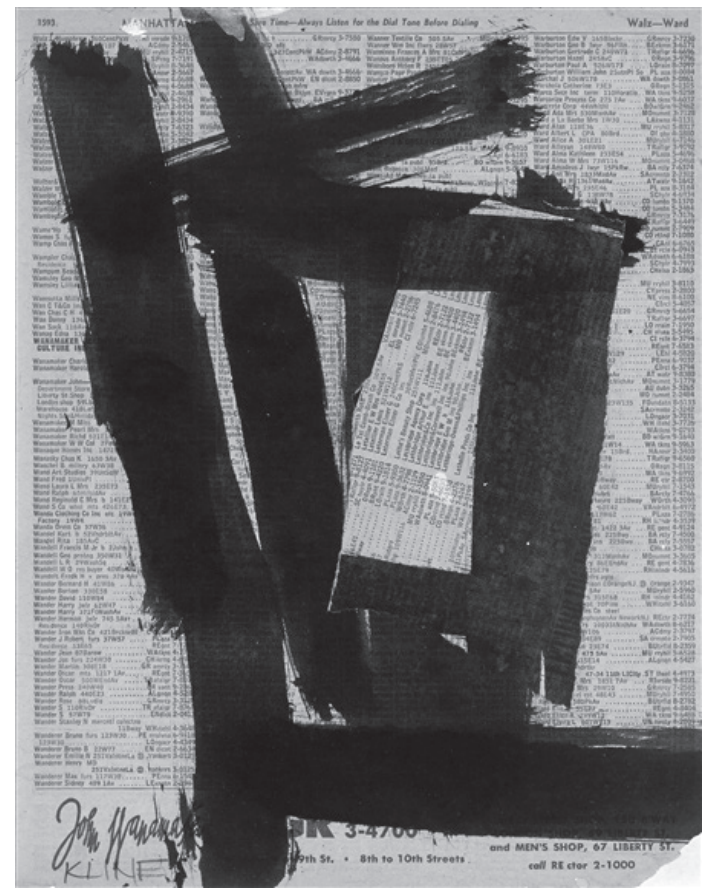

Fig. 1. Franz Kline, Untitled II, Collage y tinta sobre papel arrancado de guía telefónica y sobre cartón, 1952, 29.1 x $24.13 \mathrm{~cm}$.

combinada con el uso de las brochas habituales que se emplean para pintar en casa, herramienta empleada con asiduidad por los action painters, como Willem de Kooning, Jackson Pollock y Stewart Buettner, entre otros muchos.

Dependiendo de las intenciones ejecutivas, algunos sketch podían finalizar de una manera menos premeditada, mientras que otros requerían procesos dilatados y reflexivos en su materialización. De hecho, por ejemplo, según Stewart Buettner: "Para los pintores de acción del movimiento (Pollock, de Kooning, Kline), era más difícil concebir el punto de finalización en el cual acaba la relación del artista con el lienzo. Efectivamente, el concepto de lienzo inacabado ganó una cierta popularidad entre estos artistas. El problema parecía residir en esta cuestión: Si la pintura era el resultado de profundas emociones sentidas, ¿Cuándo pararía el pintor de sentir el lienzo?"18

Resulta evidente que desde finales de los años 40, Kline ya se había estado moviendo en la dirección de aislar la forma natural y las características de los brochazos. Si de Kooning le

csblog/post/205-1-franzkline.html. Última consulta: 05-06-2017.

18 BUETTNER, Stewart, American Art Theory 1945-1970, UMI Research Press, Ann Arbor, Michigan, 1981, p. 98. 
recomendó pasar del formato pequeño a escalas mayores, esto parece pertenecer más al ámbito de la leyenda. En cualquier caso, en 1950 ya había asumido el uso del gran formato, coincidiendo con el año de su exposición en la Charles Egan Gallery de Nueva York. Durante los años 50, ampliaría su estilo a una paleta más abierta al color. En su descripción de 1979 sobre las abstracciones cromáticas de Kline, Harry Gaugh documenta y hace hincapié en la persistencia del color a lo largo de su carrera, ya que, según él, "la alianza creativa entre (los blancos y negros de Kline) y el color ha sido pasada por alto" ${ }^{19}$. Incluso apunta que algunas de las obras sobre papel de las guías telefónicas son en color y que muchos de los negros y blancos pintados entre 1950 y 1956, cuando empezó a dar color al primer plano en las pinturas de nuevo (como lo hizo antes de 1950), fueron a menudo pinturas cromáticas sobrepintadas con blanco y negro. De hecho, a veces se dejaban pequeñas huellas de color alrededor de los bordes como claves de sus estados anteriores.

Entre otros trabajos, destacamos Herald (1953-54), caracterizado por transportar largas y estrechas proporciones de una banda, lo que podría contener las fuentes alternas de una cabecera de periódico o de una señal de calle (Herald Square) y que obviamente tuvo su preliminar en papel de libreta telefónica. Por otro lado, el óleo Zinc Door (1961) tendría su precedente en un interesante estudio de 195052 sobre este mismo soporte, donde se atisban estructuras que simulan puertas móviles hacia distintos espacios. Este tipo de acercamientos cuadrangulares, se puede apreciar en numerosas propuestas como Untitled (1951) (figura 2), donde una estructuración vertical sustenta una sección cuadrangular, siendo realizada la propuesta sobre una hoja volteada de la guía telefónica.

En muchos de estos experimentos, Kline reconoció las huellas de la imaginería: "Hay formas que son figurativas para mi, y si estas se desarrollan en una imagen figurativa...resulta correcto si así lo hacen. No tengo el sentimiento de que algo tiene que ser completamente no asociativo hasta que la presencia formal esté conformada." ${ }^{20}$ En relación con esto último, los

GAUGH, Harry F., Franz Kline: The Color Abstractions, The Phillips Collection, Washington, D.C., 1979, p. 10.

20 KLINE, Franz. En RODMAN, Selden, Conversations with Artists, Devin-Adair Co., New York, 1957, pp. 109-110. distintos bocetos en los papeles de las guías telefónicas al igual que sus posteriores materializaciones en otros formatos, de acuerdo con Andrew Graham-Dixon, aunque "son abstractos, estos parecen apuntar un fuerte sentido de lugar y, en el mejor de los casos, parecen memorias muertas de imponentes monumentos como son los puentes y los rascacielos de Nueva York. Palladio, con sus enormes y pesadas formas negras, se apresuraba en la indiferencia de algunas de las pinturas más excitantes de Kline, es como un recuerdo de Nueva York visto desde un automóvil que se apresura o por las ventanas de un tren elevado." 21

Este reduccionismo formal recuerda las prácticas realizadas en el arte japonés, ya que el método tradicional japonés "no ha sido el copiar la naturaleza como hacemos en Occidente, sino tomar solamente los elementos esenciales de la belleza que se encuentran en la naturaleza después de una larga y cuidadosa observación. Estos elementos se reproducen de un modo abstracto y simplificado hasta llegar a representar los estados de ánimo de la naturaleza. Obviamente esto exige una técnica muy perfeccionada, pero también la relación constante con la naturaleza hace a los artistas japoneses capaces de expresarla como ningún otro."22 En esta misma línea, según Chong Shan: "El valor de una pintura no está en su apariencia, sino en si; pero si es de otra manera el pintor no ha extraído la verdadera naturaleza de la imagen y no ha extraído su espíritu. Una pintura debe tener la imagen y el espíritu de un objeto que es pintado."23

Las estructuras tendentes en base a cuadraturas será una de las prácticas habituales de sus propuestas tanto en los bocetos, caso de Untitled (1950), Untitled II (1952), Untitled (1953) como en las consiguientes materializaciones sobre lienzo: Wotan (1950). Junto a esta proyección de cuadra-

21 GRAHAM-DIXON, Andrew, "ART / The master builder: Franz Kline didn't talk much about his work - one reason, perhaps, why others haven't much either. Andrew Graham-Dixon on a forgotten hero", Independent, Tuesday 12 July 1994. Web: http://www.independent. co.uk/arts-entertainment/art/art-the-master-builderfranz-kline-didnttalk-much-about-his-work-one-reason-perhaps-why-others-1413373.html. Última consulta: 13-06-2017.

22 GARCÍA GUTIÉRREZ, Fernando, Japón y Occidente. Influencias reciprocas en el arte, Ediciones Guadalquivir, Sevilla, 1990, p. 18.

23 SHAN, Chong, "Chinese Calligraphy", en Chinese Painting \& Calligraphy. 5th Century BC - 20th Century $A D$, Zhaohua Publishing House, Beijing, 1984, p. 123. 


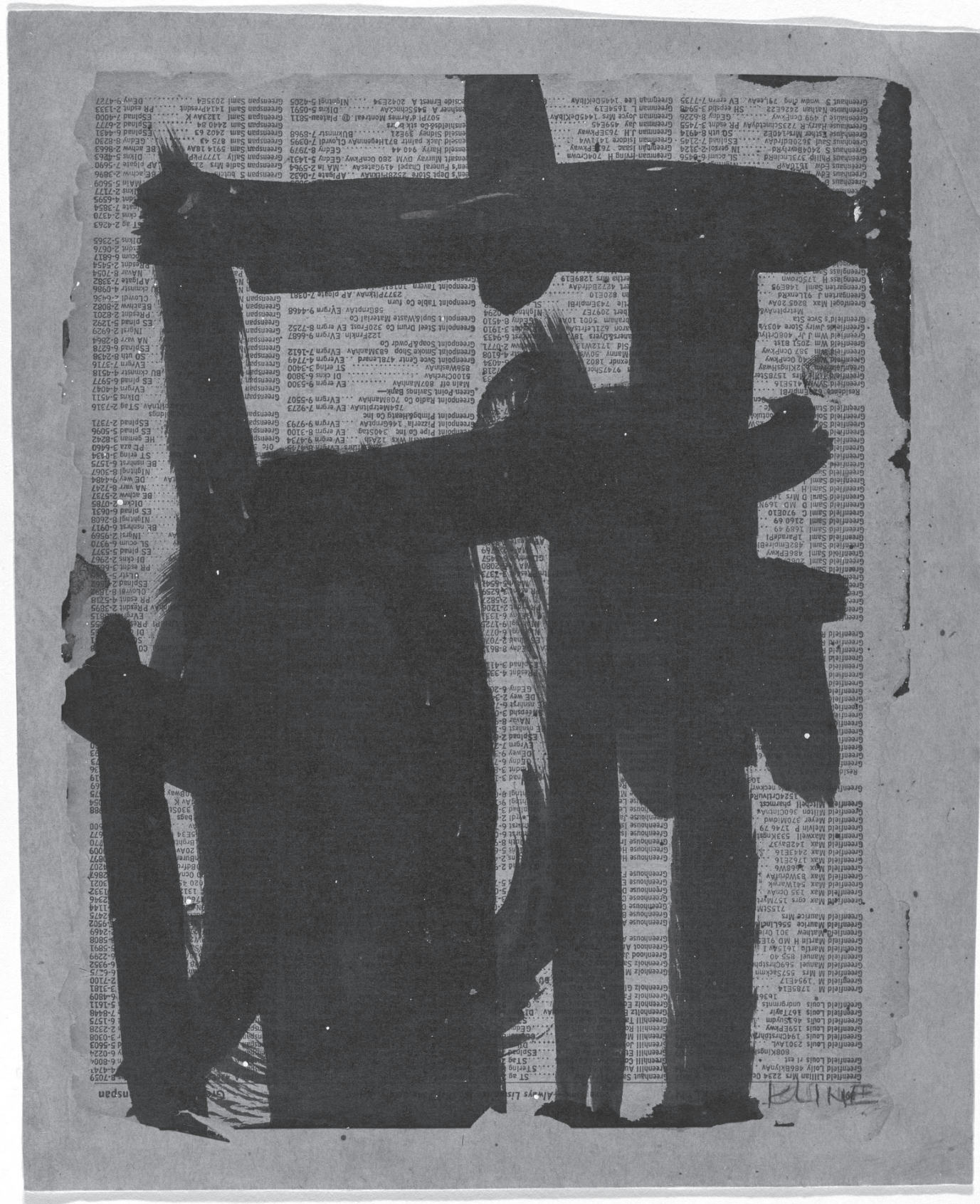

Fig. 2. Franz Kline, Untitled, Tinta sobre papel arrancado de guía telefónica y sobre cartón, 1951, 28.6 x $22.9 \mathrm{~cm}$.

turas, también se abordan manchas cromáticas que acercan su entramado hacia el triángulo, caso de Untitled (1950), tal y como se puede apreciar en Study for High Street (1950). En todas ellas, no se mantienen pautas relacionadas con una conformación equilibrada de las secciones que sustentan la composición formal interna, ya que la tendencia es jugar con diagonales que producen efectos de desestabilización visual.
La elaboración de los contundentes trazos se suelen aplicar generalmente disponiendo la hoja en sentido vertical, respetando el orden habitual de lectura de la página. No obstante, encontramos otros casos, donde la aplicación gestual se desarrolla sobre la hoja dispuesta de manera horizontal, siendo éste el caso de la anterior obra y Untitled (1953) (figura 3). Dependiendo de los intereses formales y compositivos 
que pretenda analizar, se disponían un tipo de colocaciones u otras.

Los restos de manchas procedentes de procesos de deterioro del papel como por el acto de ensuciamiento por parte del artista son rastros que se pueden identificar habitualmente, caso de Untitled (1950), donde incluso los bordes de las hojas pueden presentarse sinuosos, lo que demuestra la profunda manipulación táctil de estos materiales por parte del artista, como se puede observar en Untitled (1950), lo que no desestima ningún tipo de confrontación con el soporte material.

Si el uso del blanco y el negro se convierte en uno de los referentes habituales en sus procesos de experimentación, no podemos olvidarnos de la importancia que imprime a operaciones demarcadas única y exclusivamente por trazos de color negro. Mientras en unos cometidos el color negro ejerce un único dominio presencial, en otros ensayos se intercala la alternancia con el blanco, en constantes juegos de bipolaridad cromática, caso de Untitled (1950). Igualmente, las estructuras compositivas pueden albergar desde postulados que remiten a formas más simplistas, caso de Untitled (1950) a estructuras organizativas que se concatenan en redes de diversas líneas, tal y como se puede apreciar en Untitled (1950-52) y Untitled (1954).

\section{Los paper telephone books y el debate} generado en torno a la caligrafía japonesa

La influencia de la caligrafía ha resultado fundamental para entender el arte moderno asiático, ya que como bien comentan algunos teóricos: "La pintura oriental asiática ha llegado a su madurez en un mundo de riqueza superabundante de formas de caracteres escritos en los cuales la forma y el contenido son ambos símbolos. Esto ha empujado a ambos a un gusto y un talento por la abstracción formal, no para mencionar el elemento decorativo estilizado. Debido a las estructuras que he descrito, la caligrafía se ha vuelto un elemento resistente en la estabilidad y continuidad cultural del Asia Oriental." 24

Si en un principio, algunos estudiantes japoneses no querían considerar el Zenga como “arte", este posicionamiento se vería alterado

24 GOTZE, Heinz, Chinese and Japanese Calligrapy. Spanning Two Thousand Years, Prestel-Verlag, Munich, 1989, p. 33. por la aparición del Action Painting, el arte conceptual y otras manifestaciones propias de la modernidad tardía. Igualmente, para Stephen Addiss: "El Zenga ya ha tenido un efecto en Occidente, siendo seriamente recopilado por individuos como por museos. Ha inspirado a numerosos artistas. Franz Kline, por ejemplo, ha realizado numerosas pinturas en blanco y negro de gran formato con trazos caligráficos. Robert Motherwell recientemente rindió homenaje al Zenga en pequeñas pero intensas litografías de tres formas básicas que habían sido previamente representadas por Sengai..." 25

La caligrafía japonesa tradicional, que implica el texto con tinta (sumi) o los caracteres escritos en el papel de arroz, ha influido en muchos artistas occidentales y japoneses en épocas modernas. Algunos han incorporado la caligrafía como marcas en su trabajo, mientras que otros han sido influenciados por los ideales del Zen en base a la espontaneidad y simplicidad que la práctica implica. Muchos de los condicionantes abordados por el Expresionismo Abstracto parecen tener referencias dentro del arte asiático, caso del estilo denominado hatsu-boku, practicado por el pintor Sesshu (14201506), traduciéndose hatsu como salpicar, chapotear y boku como tinta, que sería aplicada mediante el empleo tanto de trazos muy definidos como otros estipulados por líneas curvas y más suaves. Es por este motivo por el que algunos expertos hablan de Abstract Calligra$p h y^{26}$, con el objetivo de extraer las máximas posibilidades del pincel tradicional, sin ningún interés por la legibilidad de los caracteres que transcriben. A partir de aquí, surge un grupo formado en 1950, que aglutinó a los siguientes integrantes: Ueda, Ogawa, Hidai, Shinoda y otros pintores de Tokyo, mientras que en el grupo de Kyoto destacaban Morita Shiryu y el conjunto de sus seguidores conocido como Bokujin-kai. ${ }^{27}$ En la publicación de este colec-

25 ADDISS, Stephen, The Art of Zen. Paintings and Calligraphy by Japanese Monks 1600-1925, Harry N. Abrams, Inc., New York, 1989, p. 204.

26 En relación con esta tendencia, resulta de gran interés la siguiente consulta: MEDIAVILLA, Claude; MARSHALL, Alan; STONE, Mark van; XURIGUERA, Gérard; JACKSON, Donald, Calligraphy: From Calligraphy to Abstract Painting, Scirpus Publications, Wommelgem, Belgium, 1996.

27 Morita Shiryu publicó desde 1951 a 1980 el periódico mensual Bokubi, donde se mostraron todos los trabajos relacionados con esta línea de estudio, además de importantes fuentes teóricas y documentales. Se presenta 


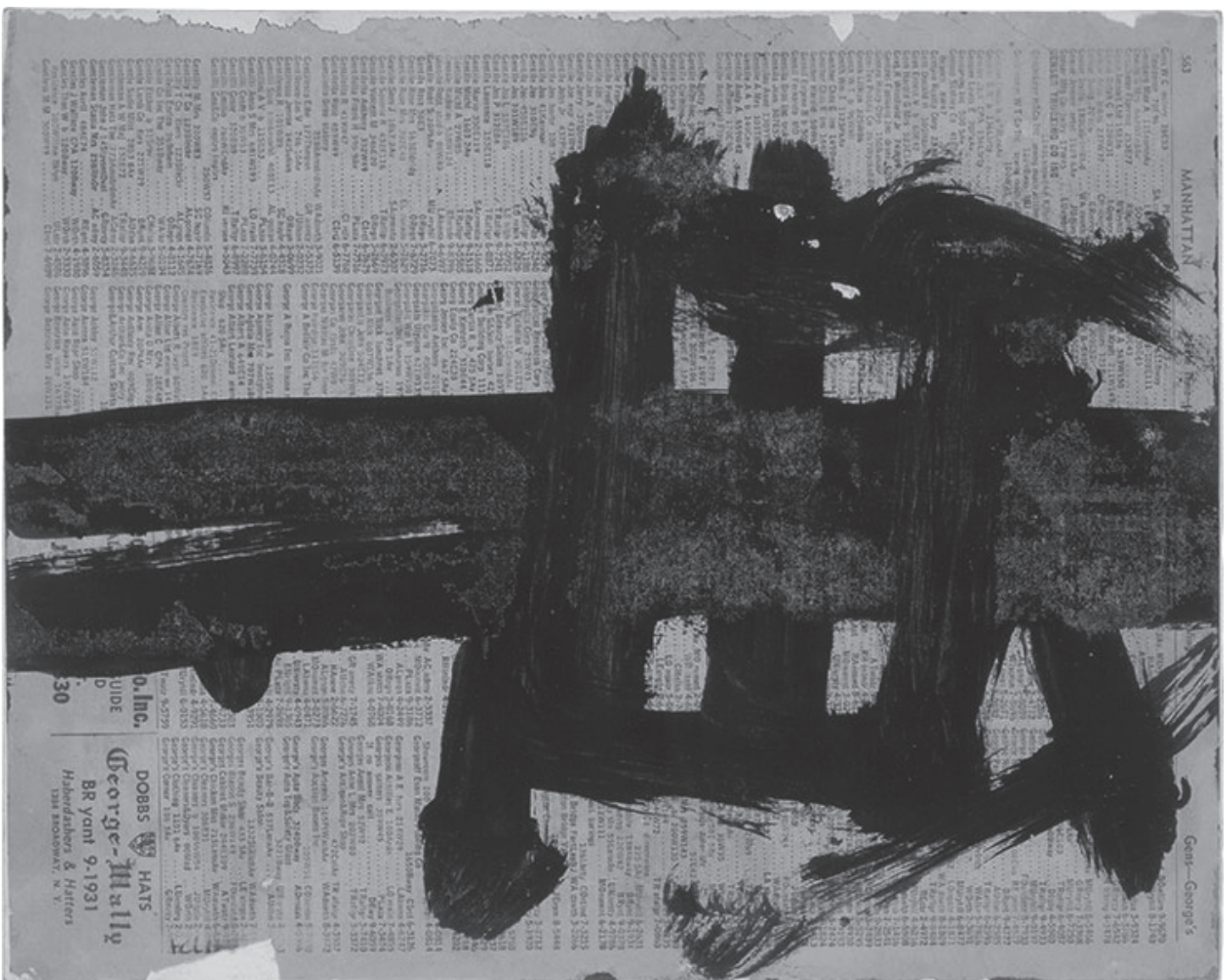

Fig. 3. Franz Kline, Untitled, Tinta y óleo sobre papel arrancado de guía telefónica y sobre cartón, 1953, 27.9 x $22.5 \mathrm{~cm}$.

tivo, tomaría también parte Hasegawa Saburo, quien publicaría obras de Kline, Tobey y Motherwell. Este autor japonés redactaría artículos de gran valor histórico en torno a la modernidad europea, introduciendo a los artistas japoneses en los valores estéticos del Surrealismo y el Expresionismo Abstracto. Además, fomentaría el potencial de la gestualidad de la caligrafía japonesa, convirtiéndose en un auténtico referente en el uso de la espontaneidad para la abstracción occidental.

Los planteamientos tanto de estos colectivos anteriores como del Expresionismo Abstracto son muy similares, al abogar ambos por la vitalidad y la espontaneidad, dejando a un lado la precisión y la elegancia; con aplicaciones rítmicas, tensiones y equilibrios ocultos; favoreciendo la insinuación y la intuición sobre

de interés la siguiente publicación: SALEL, Stephen, "Conjuring Dragons: Morita Shiry and the Globalization of Abstract Expressionism", en Abstract Expressionism, Looking East from the Far West, Honolulu Museum of Art, Honolulu, 2017. los cálculos intelectuales, en pro de la estimulación imaginativa más que de una aplicación lógica. Tal y como lo define Fernando García Gutiérrez: "Con estos puntos se intenta lograr transformación y fuerza expresiva. Estos artistas buscaron una base estabilizadora con las derivaciones del naturalismo taoísta y sintoísta, la exactitud y concisión del Zen, en los trazos rápidos y desenfadados de la caligrafía lineal y de la pintura en tinta." ${ }^{28}$

Muchas de las operaciones experimentales de Franz Kline asumen similitudes formales con la caligrafía japonesa, siendo un referente evidente de esta posible conexión formal el óleo Wotan (1950), que asume un parecido dentro del silabario japonés ${ }^{29}$ katakana con el símbolo fonético R0; Untitled (1950) (figura 4) con cinco bandas horizontales se acerca al valor fonético

28 GARCÍA GUTIÉRREZ, opus. cit., pp. 214-215.

29 El japonés tiene 2 silabarios principales conocidos como hiragana y katakana. Si este primero se usa para escribir las palabras propias del idioma japonés, el katakana se emplea para transcribir palabras importadas de otros idiomas. 
MI en la versión katakana. Por ejemplo, en Untitled (1956) (figura 5), los trazos se encorsetan en dos bandas laterales para expandirse desde un punto focal, lo que también demuestra su interés por el juego gestual y automático de líneas explayadas, aproximándonos a diferentes sílabas del hiragana. Igualmente, destaca Study for Clockface (1950), donde sus bandas negras se mueven a través del espacio del cuadro con autoridad. Resulta destacable la marcada superficie pictórica donde el negro estructura toda la composición en base a figuras primarias como el círculo abierto y el rectángulo. En general, mostraba una clara solvencia en el manejo de dichas formas básicas, que se disponían bajo una cierta correspondencia y equilibrio presencial, siendo la reciprocidad el principio que gobierna la comunidad de sus estructuras. Gaugh ${ }^{30}$ también relata que Thomas B. Hess le sugirió que la forma circular de algunos estudios sobre papel se producía al colocar el artista la tapa de un bote de pintura sobre dicho soporte. En algunos casos, el contorno del círculo podía ser demasiado extenso como para encontrar tapas de botes de este tamaño, pero tampoco sería extraño que Kline hallara algunas de este tipo.

La influencia del pensamiento y la estética oriental fue relevante para artistas y escritores como Ezra Pound, John Cage, Jack Kerouac, Allen Ginsberg, John La Farge, Arthur Wesley Dow, Morris Graves, Agnes Martin y Adrian Piper, entre otros muchos nombres dentro de una extensa lista de incorporaciones. En el caso de Kline, estas referencias con los signos caligráficos de origen japonés se remontarian incluso a finales de los años treinta, cuando el artista empieza a estudiar a fondo el arte oriental. Durante esos años, ya estaba coleccionando grabados japoneses, siendo uno de los motivos por el que posteriormente comenzarán a relacionar sus cuadros en blanco y negro con la caligrafía de dicho país. Este ejercicio comparativo no sólo se cierne a sus ejecuciones finales en los lienzos, sino que se originan directamente con las primeras propuestas procesuales sobre las páginas de guías telefónicas. No obstante, el artista rechazaría esta interpretación, ya que no aplicaba la pintura negra sobre fondo blanco, sino que sobrepintaba el blanco sobre el negro o al revés, en lo que sería una tendencia hacia un mayor interés estructural. Otro de los elementos que lo separaban de estas tesis identifi-

30 GAUGH, opus. cit., p. 93.

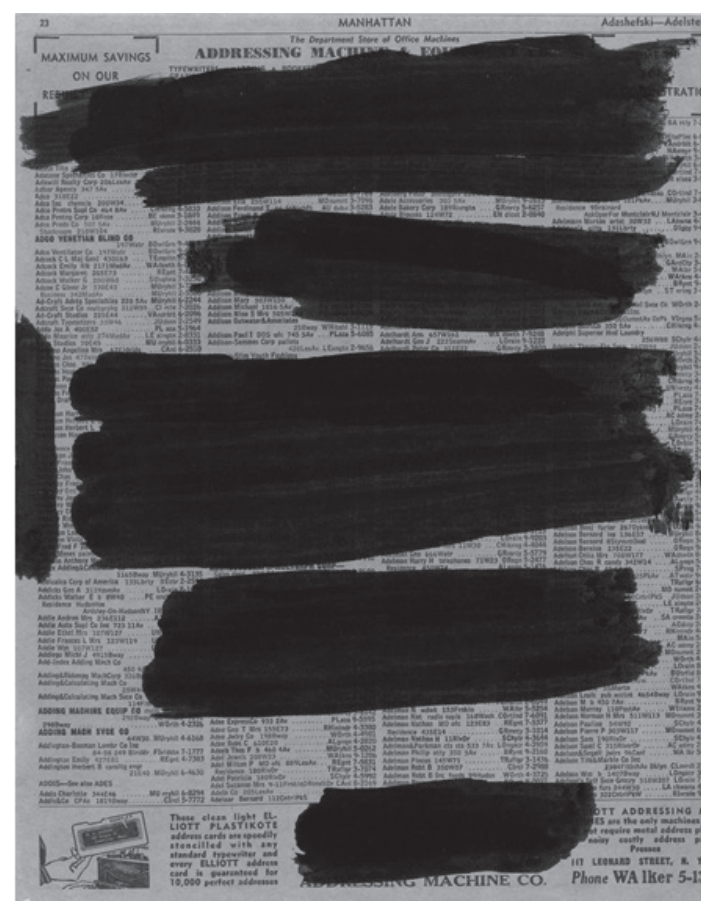

101

Fig. 4. Franz Kline, Untitled, Tinta sobre papel arrancado de guía telefónica y sobre cartón, 1950, 27.9 x $22.8 \mathrm{~cm}$.

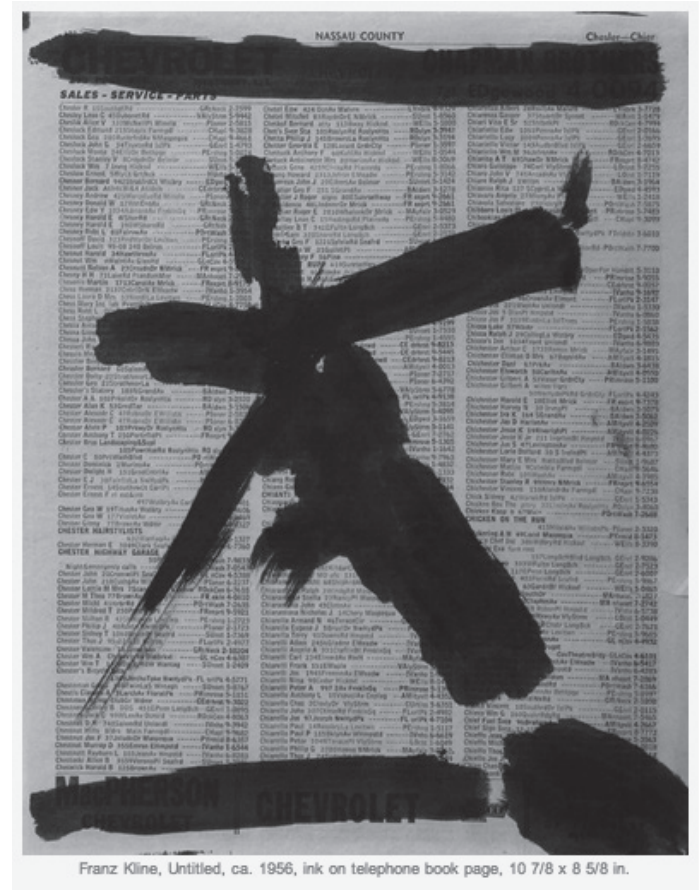

Fig. 5. Franz Kline, Untitled, Tinta sobre papel arrancado de guía telefónica y sobre cartón, 1956, 27.6 x $21.9 \mathrm{~cm}$.

cativas era que su proceso ejecutivo podía durar semanas o meses en comparación con el rápido trazado de un dibujo a la aguada sobre papel realizado en el arte asiático. El artista realizó las siguientes declaraciones a Katharine Kuh en 
1962: "En la concepción oriental, el espacio es ilimitado; no es, como el nuestro, un espacio pintado. La caligrafía es, ante todo, escritura, y yo no escribo. Algunos creen que tomo un lienzo blanco y pinto en él un signo neutro, pero no es así. Pinto tanto lo blanco como lo negro, y lo blanco es exactamente igual de importante." ${ }^{31}$ A pesar de tratarse aparentemente de trazos negros sobre fondo blanco, Kline siempre ha sostenido la igualdad de importancia entre los dos colores, configurando entre ambos volúmenes y huecos compositivos.

Los críticos han debatido durante mucho tiempo si las pinturas en blanco y negro de Kline estaban inspiradas en la caligrafía japonesa. La sugerencia partió por primera vez cuando se empiezan a examinar con detenimiento tanto sus bocetos como sus materializaciones en papel blanco y sobre lienzo, especialmente a raíz de su muestra en la Charles Egan Gallery (1950). En cualquier caso, el artista siempre lo negó, alegando que sus inspiraciones provenían de fuentes inconscientes. Cuando se le pidió que explicara el significado de su trabajo, se opuso, diciendo que quería que los espectadores sintieran los efectos de la composición sin impedimentos por la sugerencia. Enfatizó el carácter no simbólico de su obra, definiéndolo como una "experiencia de la pintura"32, lo que fue apoyado por críticos como Clement Greenberg, que se centró en la importancia de la forma abstracta en el arte, y las discusiones marginadas y provenientes de los contenidos. Por otro lado, Kline quiso poner distancia entre él y sus contemporáneos, como Mark Rothko y Barnett Newman, cuyo arte marcaba un impulso a la trascendencia. De hecho, "más que explicar o analizar el contenido de sus trabajos, Kline animaba a los espectadores simplemente a interactuar con las marcas y las mismas composiciones, no buscando simbolismo o significado, sino únicamente a interactuar con las cualidades formales del arte. Estos trabajos pretendían potenciar una singular apreciación estética en base a sus trazos personales de brocha y los espacios negativos generados alrededor. Kline sentía que el impacto emocional del trabajo podría ser experimentado enteramente a través de la delectación de estas

31 KLINE, Franz. En KUH, Katherine, The Artist's Voice: Talks With Seventeen Modern Artists, Harper \& Row, New York, 1962, p. 144.

32 KLINE, Franz. En FOSTER, Stephen C., Franz Kline and the Structure of Identity, Whitechapel Art Gallery, London; Electa, Milan, 1994, p. 36. cualidades formales e insistió que este era el aspecto más importante sobre el que se debía focalizar el estudio de sus propuestas pictóricas. A través de estas premisas personales, se convirtió en una especie de enlace entre el misticismo del Expresionismo Abstracto y el formalismo asumido por los minimalistas." 33

Por ejemplo, en la exposición celebrada en el Guggenheim de Nueva York bajo el título The Third Mind: American Artists Contemplate Asia, 1860-1989, se explora la influencia de la cultura asiática y el impacto del budismo zen, la caligrafía y la metafísica oriental en el arte americano. Alexandra Munroe, comisaria de la muestra, enlazó el Expresionismo Abstracto con la caligrafía japonesa y china, de hecho, se plantea que artistas como Robert Motherwell, Franz Kline $^{34}$ y David Smith, entre otros, encontraron inspiración en el arte de los ideogramas, en su afán por encontrar nuevos métodos compositivos y formales para la pintura. La caligrafía asiática aportaba nuevas cualidades, ritmos, juegos entre el control y el dinamismo, mediante el uso de brochazos y gestos que se incorporaban como parte del proceso ejecutivo. A pesar de que en dicha colectiva, se asumía que la pintura de estos artistas era una reminiscencia del arte y la caligrafía japonesa, igualmente en cuestiones de tamaño, audacia y sentido de la acción, ésta tomaba una dirección completamente nueva.

Según el experto y teórico Reiko Tomii: "El ensayo de catálogo de Bert Winther-Tamaki documentaba muy bien cómo el encuentro con la caligrafía asiática influyó en artistas como Kline. David Smith hizo un esfuerzo concertado para estudiarlo y más tarde negó que fuera una influencia directa." ${ }^{35}$ Jeffrey Wechsler realiza unos comentarios de gran interés en relación a las influencias orientales recibidas por los artistas expresionistas abstractas y a colofón de la anterior exposición: "sabemos por qué se puso el Kline junto al Hasegawa Saburo: se trataba de cerrar el ataúd sobre la negación de Greenberg en relación con las influencias asiáticas en el arte estadou-

33 IDEELART, opus. cit., s/n.

34 El artista iría incluido dentro de la sección Abstract Art, Calligraphy, and Metaphysics, con el trabajo titulado Mahoning (1956). También, se pusieron en contacto con el ámbito asiático trabajos de Sam Francis, Philip Guston, Isamu Noguchi, Robert Motherwell, Jackson Pollock, David Smith y Mark Tobey.

35 TOMII, Reiko; WECHSLER, Jeffrey; YOSHIMOTO, Midori; et al., "Exhibition as Proposition: Responding Critically to The Third Mind", Art Journal, vol. 68, 3 (Fall 2009), p. 36. 
nidense. Se puso un excelente texto de Robert Motherwell en donde el artista reconoce claramente la influencia de la caligrafía y otros conceptos asiáticos. El objetivo es básicamente decir adiós al rechazo asiático que emitía Greenberg, ya que era un producto del americanismo de ese período: no se podía admitir ninguna influencia extranjera, ni siquiera la influencia europea."36

También, resultan de interés, en relación con la anterior muestra, las aportaciones reflexivas de Midori Yoshimoto: "El trabajo de Kline ${ }^{37}$ estaba en la portada de su revista, Bokubi, que en la exposición es expuesto justo al lado de la obra de Kline. Algunos espectadores podrían simplemente asumir sin leer el texto de pared que Kline tenía esta revista en su biblioteca. Pero es al revés: los calígrafos japoneses de vanguardia se interesaron por el trabajo de Kline y lo vieron como la validación de nuevas direcciones en el arte japonés, como Gutai. " ${ }^{38}$ En cualquier caso, y a pesar de estas opiniones, de acuerdo a Mary A. Nicholas ${ }^{39}$, Kline argumentaba de manera constante su oposición a la comparativa que se le realizaba entre sus composiciones y la propia caligrafía oriental.

Estas supuestas similitudes formales entre las pautas caligráficas y las propuestas de Franz Kline en sus papeles de guías telefónicas conllevan una cierta complejidad a la hora de interpretar dichas composiciones, por ejemplo, para ciertos autores: "Algunas personas asumen que si no podemos leerlo, podemos apreciarlo.... Mientras el sentido de las palabras es importante para la total apreciación de la caligrafía, las palabras son secundarias para el elemento visual, por lo tanto aquellos de nosotros que no leemos chino o japonés podemos actualmente tener la mejor oportunidad de apreciar el arte como comunicación directa más allá de las palabras." ${ }^{40}$

Por otro lado, en opinión de Claude Mediavilla: "Escritura y pintura son aparentemente dos ámbitos diferenciados. Sin embargo, en su origen, la escritura pictográfica no es más que una pura representación del mundo, es decir, los signos que la componen permanecen muy

\footnotetext{
$36 \quad$ Ibid, p. 37.

37 Se refiere al cuadro titulado Clock Face (1951), que aparece en el número 1 de la portada del periódico mensual Bokubi.

38 TOMII, Reiko; WECHSLER, Jeffrey; YOSHIMOTO, Midori; et al., opus. cit., p. 42.

39 NICHOLAS, Mary A., "We Were Born to Make Fairytales Come True: Reinterpreting Political Texts in Unofficial Soviet Art", Canadian Slavonic Papers, 53, 2-4 (Jun-Dec 2011), p. 351.

40 ADDISS, opus. cit., p. 19.
}

cercanos a la representación pictórica. Poco a poco, estos signos fueron evolucionando hacia la abstracción alfabética; por tanto, existe una relación esencial entre escritura y pintura." ${ }^{41}$

Se podrían generar otras muchas conexiones hipotéticas, tal y como lo postula Ana Crespo García $^{42}$, artistas como Pollock, Kline, Gottlieb, aunque beben de las fuentes del Zen, parecen estar más relacionados con los pintores taoístas llamados ipin. En cualquier caso, en los trabajos de Kline hay un acercamiento formal y compositivo al fenómeno Wabi, donde se aprecia una estética de la simplicidad, eliminando lo ficticio que se encubre todo, buscando de esta manera un vínculo directo con el interior de la forma o el objeto. Asimismo, podríamos realizar una conexión con el estilo Sabi, como sentido de soledad mediante la aplicación de trazos solitarios en medio de grandes espacios vacíos, que están llenos de significado; e incluso con el Shibumi a modo de trazos inacabados y desenfadados de la pintura. Según Hisamatsu Shin-ichi: "Siempre es la interioridad sin forma la que, en cada ocasión, expresa de un modo creativo a sí mismo. Por tanto, la cuestión no está en ver en qué medida se puede expresar algo de un modo realista o impresionista. Más bien la cuestión está en ver cómo la interioridad misma puede ser expresada de un modo libre y creativo." ${ }^{43}$

\section{Conclusiones}

Los estudios referentes a los trabajos experimentales de Franz Kline sobre páginas de guías telefónicas son escasos y reducidos, ya que históricamente la orientación deductiva se ha enfocado de manera constante sobre propuestas materializadas en papel blanco y lienzos. Pero, estos primeros procesos de tanteo resultan fundamentales para entender la gestación pictórica de este expresionista abstracto. Sobre estos soportes ideaba toda una serie de prácticas que englobaban la colocación de diferentes objetos que eran siluetados; experimentaciones con grosores, coágulos pictóricos y adhesión de elementos externos (suciedad, manchas de grasa y todo tipo de

41 MEDIAVILLA, Claude, Caligrafía. Del signo caligráfico a la pintura abstracta, Campgráfic Editors, Valencia, 2005, p. 292.

42 CRESPO, Ana, La Realidad y la Mirada. El Zen en el Arte Contemporáneo, Mandala Ediciones, Madrid, 1997, p. 102.

43 SHIN-ICHI, Hisamatsu, Zen and the Fine Arts, Kodansha International Ltd., Tokyo, 1971, p. 19. 
sustancias no artísticas). La toma de contacto con estas intrusiones físicas le permitía ahondar en nuevas oportunidades reflexivas sobre aspectos estructurales, compositivos y formales.

Se sintió atraído por este soporte no sólo debido a su rápida y cercana disponibilidad, sino también motivado por sus fondos amarillentos, lo que le acercaba visualmente a los fundamentos pigmentados de las sedas empleadas en la cultura asiática. Asimismo, las columnas de nombres y sus correspondientes extrapolaciones numéricas llegaban a servir como estructuraciones para la extensión de todo tipo de trazos de mayor o menor envergadura y anular cualquier recesión de perspectiva ilusoria.

El proceso conceptual y ejecutivo de la mayoría de las obras pictóricas del artista tanto sobre papel como sobre lienzo remite a estos medios, convirtiéndose en muchos casos en meras prolongaciones a partir del original experimental. Esta práctica demuestra que muchos trabajos se comportan como pautas premeditadas y de una cargada construcción, sin dejarse llevar por la deriva crítica que habían tildado todos sus trabajos como gestuales e instintivos. En base a esta metodología, investigó principalmente sobre la consistencia del negro y el blanco en sus ulteriores materializaciones, no obstante, casi todas las propuestas sobre papeles de guías telefónicas fueron desarrolladas en negro tinta y óleo; mientras intercalaba de manera paralela propuestas sustentadas en los collages.

Su articulación compositiva y lineal generó en su época un debate que se podría alargar hasta la actualidad, debido a las numerosas correspondencias visuales con la caligrafía japonesa e incluso en base a su supuesta conexión con la $A b s-$ tract Calligraphy. Esta controversia se remonta a finales de los años treinta, cuando el artista empieza a estudiar a fondo el arte oriental. Durante esos años, ya estaba coleccionando grabados japoneses, siendo uno de los motivos por el que se comienza a relacionar sus cuadros en blanco y negro con esta dinámica estilística. No obstante, el tema cobrará mayor intensidad a partir de las primeras prácticas ejecutadas sobre el papel de guías telefónicas y especialmente a raíz de su exposición en la Charles Egan Gallery (1950).

Resulta evidente que se dan numerosas interpelaciones visuales con el silabario japonés, además de su amplio conocimiento del medio, siendo incluso su obra un referente para numerosos artistas japoneses modernos. Esta influencia oriental en la obra del pintor norteamericano vendría defendida por distintos expertos como Alexandra Munroe, comisaria de la muestra The Third Mind: American Artists Contemplate Asia, 1860-1989, así como por Bert Winther-Tamaki y Jeffrey Wechsler, entre otros. Pero a pesar de estas redes conectivas, se debe hacer constar la negativa por parte del artista a tal tipo de compulsaciones, tal y como es defendido por Clement Greenberg y Mary A. Nicholas, basándose especialmente en que el espectador no debía hallar ningún vector simbólico, sino únicamente sentir el efecto de la percepción sin ningún tipo de sugerencia, interactuando con las propias cualidades formales del trabajo. 